\title{
An update of molecular pathology of bone tumors. Lessons learned from investigating samples by next generation sequencing
}

\author{
Daniel Baumhoer ${ }^{1}$, Fernanda Amary ${ }^{2,3}$ Adrienne M. Flanagan ${ }^{2,3}$
}

${ }^{1}$ Bone Tumour Reference Centre, Institute of Pathology, University Hospital Basel, University of Basel, Basel, Switzerland

${ }^{2}$ Department of Pathology, The Royal National Orthopaedic Hospital, Stanmore, Middlesex, United Kingdom

${ }^{3}$ Department of Pathology, Cancer Institute, University College London, London, United Kingdom

Correspondence

Adrienne M. Flanagan, Department of Pathology, The Royal National Orthopaedic Hospital, Stanmore, Middlesex, United Kingdom.

Email: a.flanagan@ucl.ac.uk

Funding information

National Institute for Health Research;

Rosetrees Trust; Skeletal Cancer Action Trust UK; The Royal National Orthopaedic Hospital NHS Trust; UCL Experimental Cancer Centre; UCLH Biomedical Research Centre

\begin{abstract}
The last decade has seen the majority of primary bone tumor subtypes become defined by molecular genetic alteration. Examples include giant cell tumour of bone (H3F3A p.G34W), chondroblastoma (H3F3B p.K36M), mesenchymal chondrosarcoma (HEY1-NCOA2), chondromyxoid fibroma (GRM1 rearrangements), aneurysmal bone cyst (USP6 rearrangements), osteoblastoma/osteoid osteoma (FOS/FOSB rearrangements), and synovial chondromatosis (FN1-ACVR2A and ACVR2A$F N 1$ ). All such alterations are mutually exclusive. Many of these have been translated into clinical service using immunohistochemistry or FISH. $60 \%$ of central chondrosarcoma is characterised by either isocitrate dehydrogenase (IDH) 1 or IDH2 mutations distinguishing them from other cartilaginous tumours. In contrast, recurrent alterations which are clinically helpful have not been found in high grade osteosarcoma. High throughput next generation sequencing has also proved valuable in identifying germ line alterations in a significant proportion of young patients with primary malignant bone tumors. These findings will play an increasing role in reaching a diagnosis and in patient management.
\end{abstract}

KEYWORDS

bone tumor, FISH, genomics, mutations, next generation sequencing, sarcoma

\section{1 | INTRODUCTION}

Massive parallel sequencing of primary bone tumors has revealed the full spectrum of driver gene alterations including single nucleotide variants (SNVs), somatic copy number variants, fusion genes, and more complex alterations such as chromothripsis. Many of these tumors can now be classified at least superficially on the basis of highly recurrent and specific driver events, for example, the majority of osteosarcoma can be distinguished from chondrosarcoma on the basis of IDH1/2 mutations, and/or COL2A1 mutations in the latter. The systematic and comprehensive molecular analysis of these groups of tumors was largely but not exclusively achieved through the International Cancer Genome Consortium and demonstrates the benefit of large multi-institute collaborations when studying rare tumor types. Collectively, the genetic profiling of primary bone tumors has transformed the ability of surgical pathologists to deliver diagnoses more reproducibly and accurately, particularly in histologically challenging cases. This provides clinicians with greater confidence when considering treatment options. Indeed, only a few subtypes of bone tumors remain uncharacterized at a genomic level, such as sporadic cases of osteofibrous dysplasia and adamantinoma. However, there is still much to be learnt as the presence of genetic alterations does not always allow the separation of benign from malignant forms of a specific tumor type: for instance, detection of isocitrate dehydrogenase type 1/2 mutations in central cartilaginous tumors, H3F3B p.G34 mutants in giant cell tumor (GCT) of bone, and FN1-ACVR2A and ACVR2A-FN1 rearrangements in synovial chondromatosis occur in both the benign and malignant forms of these neoplasms.

Large-scale sequencing studies of tumor and constitutional DNA has in some cases led to the identification of new targets for personalized treatment approaches. Good examples include the treatment of GCTs of bone with monoclonal antibodies against Receptor activator of nuclear factor kappa-B ligand (RANKL), and the aggressive form of tenosynovial GCT with CSF1 receptor inhibitors.

Despite all of the advances, there is no laboratory test that is entirely sensitive or specific for a tumor, underscoring the need to 

interpret all molecular pathology results in the context of the histology, clinical and familial history, and the relevant medical imaging.

\section{2 | BONE-FORMING TUMORS}

\subsection{Osteoid osteoma and osteoblastoma}

According to the current World Health Organization classification of bone tumors, osteoid osteoma and osteoblastoma are regarded as separate entities within the spectrum of benign bone-forming lesions. Arbitrarily divided by size (below or above $2 \mathrm{~cm}$ in diameter), clinical and radiological features, albeit both tumors exhibit a nearly identical histology. Osteoid osteomas have a predilection for the cortex of long tubular bones but can occur anywhere in the skeleton. Osteoblastomas most commonly develop in the posterior elements of the spinal vertebra and are regarded as tumors of intermediate category (locally aggressive). Both lesions usually affect children and young adults. They do not transform into high-grade tumors. One of the most challenging tasks in diagnostic bone tumor pathology is to distinguish osteoblastoma from osteoblastoma-like osteosarcoma, especially on core biopsies.

Until recently, molecular data on osteoid osteoma and osteoblastoma were scarce. ${ }^{1,2}$ However, analysis of whole genome and RNAsequencing of five osteoblastomas and one osteoid osteoma revealed that all tumors showed an oncogenic structural rearrangement in the AP-1 transcription factor, either FOS on chromosome 14, or, in one case, its paralogue FOSB on chromosome $19 .{ }^{3}$ Notably, the previously reported loss of $22 q$ was not detected. ${ }^{1,2}$ Otherwise, the genomes revealed few and insignificant alterations in terms of SNVs and copy number aberrations. ${ }^{3}$

Remarkably, the FOS break points were all exonic, residing within a narrow genomic locus of exon 4 , and the rearrangements included both interchromosomal and intrachromosomal events. Notably, the rearrangements did not involve the coding sequence of other genes (KIAA1199, MYO1B, and ANK) and in the two remaining cases the fusion partner did not lie within a gene. Indeed, the vast majority of cases with FOS rearrangements that were detected by fluorescence in situ hybridization (FISH) were strongly immunoreactive for FOS using an antibody against the $\mathrm{N}$ terminus. ${ }^{3}$

The FOSB rearrangement, identified in the one case sequenced, revealed that the FOSB fusion gene would be brought under the control of the PPP1R1O promoter through an in-frame fusion of PPP1R1O to $F O S B$ in exon 1. Similar structural alterations involving the same region of exon 1 have been reported in vascular tumors that can also develop in bone, and include pseudomyogenic hemangioendothelioma and epithelioid hemangioma. ${ }^{46}$

Distinguishing osteoblastoma from osteosarcoma is clinically important: 183 osteosarcomas, 97 of which exhibited an osteoblastic phenotype, were analyzed for FOS expression by immunohistochemistry, and only 1 revealed positivity that was equivalent to the strong expression seen in osteoblastomas. Furthermore, FOS and FOSB genetic alterations appear to be highly specific for osteoid osteoma and osteoblastoma as analysis of the genomes of 55 osteosarcomas, revealed no FOS rearrangement. ${ }^{7,8}$ Taken together, these data show that osteoid osteomas and osteoblastomas are defined by alterations in FOS and, rarely, FOSB and that both tumors types are driven by the same genomic events. Taking into account their similar histology, these tumors most likely represent the same disease with different clinical and radiological presentations. Finally, immunohistochemistry for FOS is a simple method for screening equivocal cases for FOS rearrangement and can be used as an axillary diagnostic test (Figure 1).

\section{2 | Fibrous dysplasia}

Fibrous dysplasia is a fibro-osseous lesion: it is a skeletal anomaly caused by postzygotic missense mutations in GNAS which encode the activating alpha subunit of the stimulatory $G$-protein. It can involve single (monostotic) or multiple bones (polyostotic) and occurs alongside a range of endocrinopathies, and skin lesions such as McCuneAlbright syndrome. ${ }^{9}$ Mazabraud syndrome is defined as fibrous dysplasia and soft tissue myxoma(s). ${ }^{10}$

The GNAS mutations are most commonly involve codons 201 of exon 8 (95\%, mainly p.R201H and p.R201C) and 227 of exon 9 (5\%, Q227L). ${ }^{11,12}$ These mutations can also be identified in the so-called liposclerosing myxofibrous tumors indicating that this lesion represents a regressive form of fibrous dysplasia. ${ }^{13}$ Exceptionally sarcomatous transformation, in the form of osteosarcoma, chondrosarcoma, and an undifferentiated spindle cell sarcoma, may occur in fibrous dysplasia.

\section{3 | OSTEOSARCOMA}

Osteosarcoma is the most common primary malignant tumor of bone, generally affecting the metaphyses of long bones. It has a bimodal age distribution with the majority of cases arising in children and adolescence younger than 20 years. Aggressive high-grade tumors, represented by highly variable histological features, account for approximately $90 \%$ of osteosarcomas and are treated with neoadjuvant chemotherapy to address systemic spread that may be present at the time of diagnosis. Despite the multimodal chemotherapy, $30 \%$ $40 \%$ of patients today still succumb to their disease, mainly due to refractory and/or recurrent disease.

Ten percent of osteosarcomas are classified as low and intermediate grade, namely parosteal, periosteal, and low-grade central osteosarcoma, and are generally not treated with chemotherapy. Parosteal and low-grade central osteosarcomas represent subtypes with an indolent clinical course and both tumors can generally be cured by resection with clear margins but share the risk of transformation into a high-grade tumor, sometimes decades after the initial presentation. There is a high prevalence of MDM2 (and CDK4) amplifications in both parosteal and central low-grade osteosarcoma ( $85 \%$ of parosteal and $25 \%-30 \%$ of central low-grade osteosarcoma, respectively), which can be exploited diagnostically using FISH. MDM2 immunohistochemistry is sensitive but lacks specificity. Roughly, $10 \%$ of conventional highgrade osteosarcomas also harbor MDM2 amplification suggesting that they may have arisen from a preexisting low-grade tumor. Recently, a single study reported that five of nine cases of parosteal osteosarcoma harbor a GNAS mutation in addition to MDM2 amplification. ${ }^{14}$ 


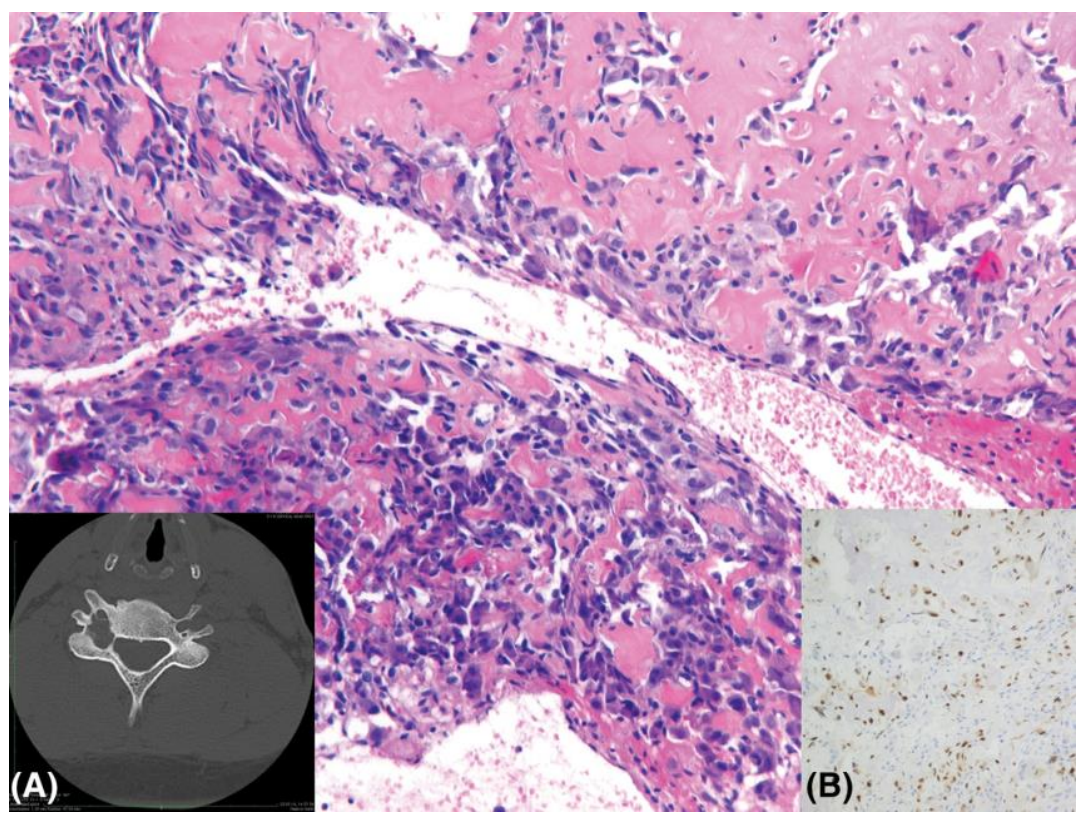

FIGURE 1 Photomicrograph of hematoxylin and eosin-stained section showing the characteristic features of an osteoblastoma. The inset on the $\mathrm{X}$-ray shows a lytic lesion in the posterior element of the spinal vertebra, and the immuno-stained section shows FOS nuclear positivity of the osteoblastic cells

This was surprising as GNAS mutations until that point, were considered to be specific for fibrous dysplasia (see above) and furthermore the recurrent SNVs in fibrous dysplasia were considered to be mutually exclusive with MDM2 amplification. This prompted a follow-up study of 97 osteosarcoma samples, 97 samples including 62 parosteal osteosarcomas and 24 low-grade osteosarcomas which failed to reveal GNAS alterations. Our results supported the previous observations that GNAS mutations are highly specific for fibrous dysplasia and not detectedin parosteal osteosarcoma. ${ }^{15}$

Despite substantial research efforts, in the majority of cases the cause of osteosarcoma is not known (see below), and the diagnosis, subtyping, and grading remain defined by morphology alone. There are no recurrent genetic alterations or molecular profiles linking the prognosis of patients or their response to chemotherapy (other than the presence of MDM2 amplification, see above), and notably survival rates have not improved significantly over the last three decades.

\section{4 | CAUSE OF OSTEOSARCOMA}

\subsection{Germ line alterations}

It is reported that $20 \%$ of patients under the age of 25 presenting with osteosarcoma have a germ line alteration predisposing them to the disease. ${ }^{16}$ The most common germ line-mutated genes in osteosarcoma are TP53 and RB1, and less commonly the RECQ helicases (RECQL2: Werner syndrome; RECQL3: Bloom syndrome; RECQL4: Rothmund-Thomson syndrome).

Other causes include ionizing radiation 7 : Pagetic bone disease associated with SQSTM1 mutations detected in $20 \%-50 \%$ of familial and $10 \%-20 \%$ of sporadic cases, in addition to mutations in TNFRSF11A (RANK) and VCP17,18; and bone infarct occurring in
Hardcastle syndrome diaphyseal medullary stenosis which is inherited as an autosomal dominant trait. ${ }^{19}$

\section{2 | Somatic alterations in osteosarcoma}

High throughput next generation sequencing technology has confirmed that osteosarcoma exhibits chromosomal instability characterized by multiple complex rearrangements, and that the number of SNV is relatively low compared to many cancers of adulthood. In 2011, chromothripsis was described in osteosarcoma and provided for the first time an explanation for the genomic complexity of this tumor type. ${ }^{7}$

\section{3 | Cancer drivergenes}

As many as 67 different cancer genes, with structural variants being the most common source of mutation, have been reported in osteosarcoma: the most common being alterations in TP53 which have been reported in as many as $88 \%$ of cases. Other genes and/or signaling pathways include MYC, PTEN, ATRX, CDKN2A, PI3K/mTOR, IGF, FGF, RUNX2, VEGFA, and E2F3.7,20 Although there have been attempts to correlate specific somatic copy number alterations and the amount of chromosomal complexity with outcome and/or response to chemotherapy none have been found superior to the histologically assessed response to treatment.

Subgroups of osteosarcomas have also been identified as harboring recurrent alterations that are potentially actionable including FGFR1 amplification $18.5 \%$ of osteosarcomas that do not respond to chemotherapy, ${ }^{21}$ and alterations in the IGF1R signaling pathway in up to $14 \%$ of high-grade osteosarcoma. ${ }^{7}$ These findings require validation in larger cohorts, and the clinical impact is tested by stratifying patients in clinical trials. 
5

This is a diverse group of tumors exhibiting features of either or both bone and cartilage differentiation but all are linked through the presence of conspicuous numbers of large osteoclast-like cells containing up to 100 nuclei. Despite these multinucleate cells being the most conspicuous cell type, it had been accepted for some time that the stromal population represents the neoplastic component. However, it has only been with the advent of molecular analysis that this has been shown definitively. ${ }^{22}$ Notably, two of the three epiphysealbased primary bone tumors-GCT of bone and chondroblastomaare osteoclast-rich: the third epiphyseal-based tumor is clear cell chondrosarcoma (see below). Remarkably, all three tumor types have been reported to harbor SNV in one of two genes, H3F3A or H3F3B, encoding the replication-independent histone 3.3. These two genes are found on chromosomes 1 and 17, respectively, but encode an identical protein. ${ }^{22}$

\section{6 | GCT OF BONE}

GCT of bone is a locally aggressive tumor with a predilection to the subarticular (epiphyseal) region of long bones. GCTs occasionally metastasize to the lung but the metastases retain the original histological features and are usually slow-growing with some cases even undergoing regression. Virtually all GCTs (96\%) harbor a H3F3 mutation which is restricted to $H 3 F 3 A$ involving specifically Glycine 34, with G34W (p.Gly34Trp [p.G34W]) accounting for the vast majority of the variants and G34L (p.Gly34Lys [p.G34L]) for a small minority. ${ }^{23,24}$ Detection of the p.G34W mutation in the nuclei of the mononuclear cells by immunohistochemistry definitively showed that this was the neoplastic cell: the antibody is highly specific and sensitive and is used for diagnostic purposes (Figure 2). ${ }^{23}$ Apart from this H3F3 driver gene mutation in GCT, there was a relatively low somatic mutation burden and copy number, and rearrangement analysis showed that tumors were diploid overall, with a paucity of structural changes. $^{22}$

Ninety nine giant cell granulomas of the jaw have been assessed for the expression of the H3.3 p.G34W mutant protein, but to date no case with immunoreactivity has been identified. ${ }^{23,24}$ This argues, until proven otherwise, that giant cell granulomas of the jaw are not only morphologically but also genetically distinct from GCT.

GCTs rarely occur in the immature skeleton, but in such circumstances they may be sited in the metaphyseal region. The identification of the H3.3 p.G34 mutant variants in such osteoclast-rich tumors argues that such neoplasms represent conventional GCT and should be diagnosed and treated as such. ${ }^{23}$

\section{I SYNDROMES INVOLVING GCT OFBONE}

Recently, a new cancer syndrome has been described involving pheochromocytomas, paragangliomas, and GCT caused by a postzygotic
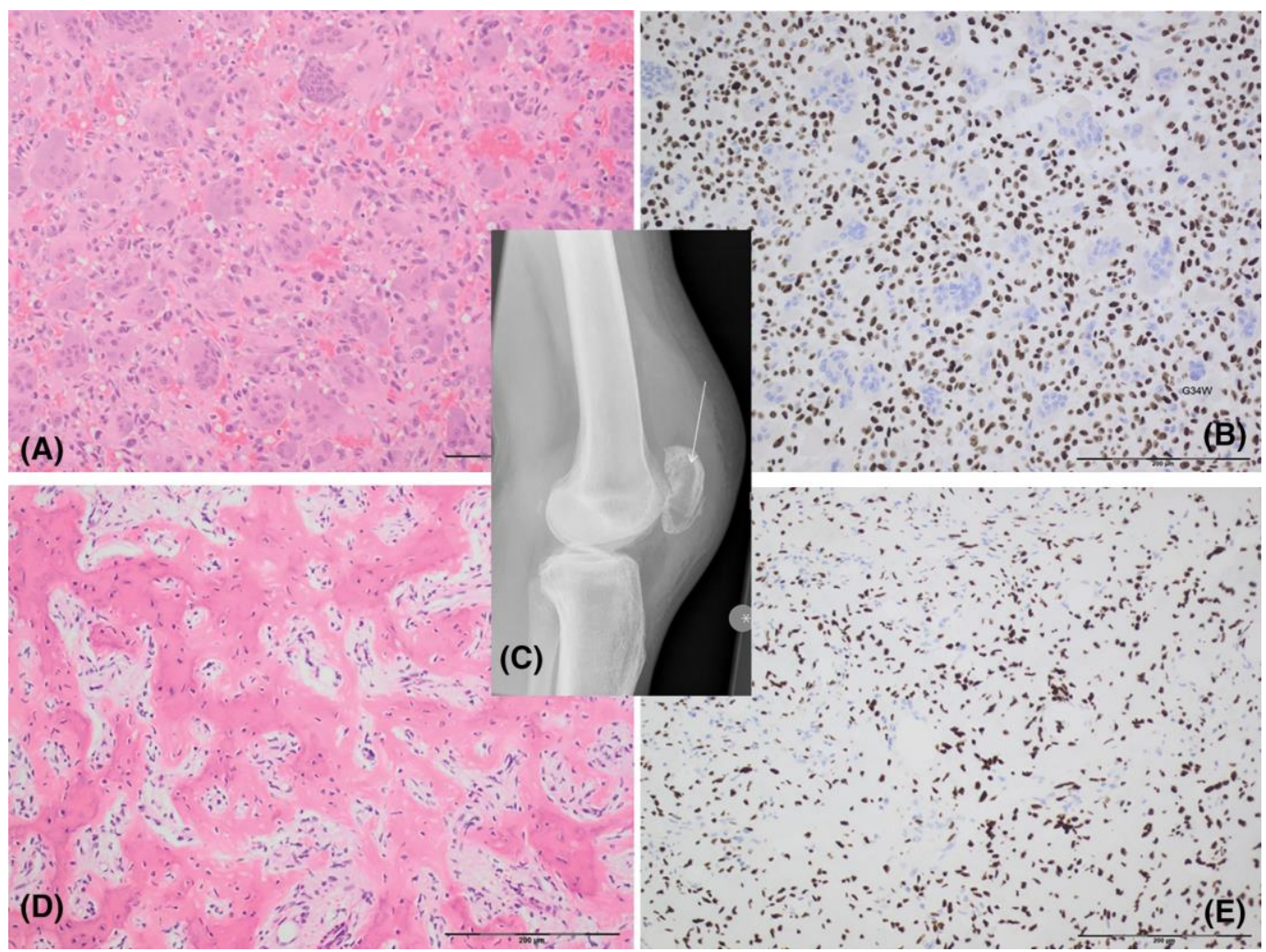

FIGURE 2 Photomicrographs of the biopsy specimen showing a conventional giant cell tumor of bone (A) with diffuse H3.3 G34W expression (B). Note that the expression is restricted to the tumor stromal cells, and it does not decorate the osteoclast-like giant cells. C, Inset of a lateral radiograph showing the lytic tumor in the patella. $D$, A post-denosumab treatment specimen showing ossification of the matrix with absence of osteoclast-like giant cells. E, H3.3 G34W expression is retained in the tumor cells 
histone 3.3 G34W mutation. ${ }^{25}$ Histologically, the GCT appears identical to the sporadicvariant.

Familial clustering has been described in Pagetic bone disease, particularly in the early onset form (see above) which can be multifocal, in individuals with GCT. Germ line missense mutations in $2810 C>G$ ( $p$. Pro937Arg) in the zinc finger protein 687 gene (ZNF687) have been found to be a familial monogenic cause of this phenotype and consistent with the autosomal-dominant inheritance pattern of the disease. These ZNF687 mutations are mutually exclusive of other genes known to be associated with a Pagetic-related syndrome (see above). ${ }^{25}$

\section{8 | CELL LINEAGE OF GCT OF BONE}

The mononuclear neoplastic cell in the GCT has been considered for some time to be of osteoblastic lineage. This view was based on the observation that although bone formation is not common, it can be extensive in a small numbers of cases; furthermore, these cells express osteoblastic markers. ${ }^{26}$ However, the most definitive evidence to date is gleaned from research published nearly 20 years ago which showed that osteoclast formation is RANKL-dependent, a molecule produced by osteoblastic cells, among others. ${ }^{27}$ This led to the development of denosumab, a monoclonal antibody targeting RANKL, which has proven useful as an adjuvant treatment of GCT. ${ }^{28}$ The finding that treatment of GCT with denosumab results in almost total depletion of osteoclast-like giant cells and the maturation of the neoplastic mutant cells, which is seen as the formation of new bone demonstrates unequivocally that the neoplastic cells are of osteoblastic lineage (Figure 2). This also reveals that osteoclasts curb bone formation. The specific molecules responsible for this have not been characterized in GCT so far, although candidates include those implicated in the reverse coupling of bone formation and resorption described in the literature. ${ }^{29}$ Most recently, there is evidence that RANK secreted by osteoclasts act to suppress bone formation by reverse signaling through osteoblastic RANKL. ${ }^{30}$

\section{9 | MALIGNANT GCT OF BONE}

Malignancy in GCT is rare but well described. In our experience, such tumors which can be difficult to distinguish from telangiectatic osteosarcoma are characterized by a H3F3A G34 mutation. There appears to be a wide variation of biological behavior in cases of malignant GCT. However, with the ability to identify a H3F3A mutation, it will be easier to distinguish these cases from other bone malignancies and generate, with time, a larger cohort of patients with such tumors, permitting a better knowledge of the disease. ${ }^{23}$

\section{0 | CHONDROBLASTOMA}

This nonconventional benign cartilaginous tumor has histological, clinical, and radiological features overlapping with those of GCT. However, the stromal cells exhibit a chondroblastic phenotype, as seen as (osteo-)chondroid matrix deposition, and the tumor presents most commonly in the immature skeleton although not exclusively. The majority is treated successfully with curettage. It very rarely metastasizes-a benign metastasizing chondroblastoma-but does not transform into a high-grade tumor.

Similar to GCT, virtually all chondroblastomas harbor a H3F3 mutation. However, the mutation is confined to p.K36 and is always substituted for a methionine. Furthermore, although there is a clear preference for the mutations occurring in $\mathrm{H} 3 \mathrm{~F} 3 \mathrm{~B}$, although occasionally they also are found in $H 3 F 3 A .{ }^{22}$

Gene expression of H3F3A and H3F3B does not distinguish between GCT (H3F3A G34W mutant) and chondroblastoma (H3F3B K36M mutant). Interestingly, different expression patterns of the two genes have been reported during embryonic and postnatal development in both normal murine and human tissues, suggesting that temporal differences may account for the activity of the two genes. ${ }^{31,32}$ H3F3A p.K27M and p.G34R/V mutations also occur in childhood brain tumors, but histone 3.3 mutations appear to be specific to certain tumor types, indicating distinct functions of histone 3.3 residues, mutations, and genes. ${ }^{33,34}$

Detection of the p.K36M in the H3F3A or the H3F3B genes is diagnostic and is best sought using immunohistochemistry as immunoreactivity in even a few cells can clinch the diagnosis (Figure 3). ${ }^{35}$ As in GCT, the neoplastic cell in chondroblastoma is the stromal mononuclear cell and not the osteoclast-like giant cell or its precursor.

Chondroblastoma in the jaw and skull bones is exceptionally rare, and to date no case with a H3.3 p.K36M mutation has been identified raising the question as to whether this tumor really occurs at this site. The analysis of a large set of such tumors will be necessary to answer this question.

The H3.3 p.K36M mutation is mutually exclusive of genetic alterations identified in other tumors which could be considered in the differential diagnosis.

\section{ANEURYSMAL BONE CYST (ABC)}

This is a benign locally aggressive osteoclast-rich tumor occurring in any bone, including the vertebral bodies. It arises in the metaphysis of long bones but may extend to the subarticular region. Approximately, $75 \%$ of cases harbor a balanced chromosomal translocation involving USP6 gene, sited in chromosome $17 \mathrm{p} 13$, with a variety of fusion partners including CDH11, ZNF9, COL1A1, TRAP150, OMD, RUNX2, and CTNNB1. ${ }^{36-39}$ As with other osteoclast-rich tumors, it is the mononuclear spindle cells that harbor the genetic alteration. ${ }^{39}$ The USP6 gene rearrangement acts as an oncogene and brings about alteration of cell migration and cytokinesis. It is important to distinguish this tumor from secondary aneurysmal cystic change "secondary ABC" associated with other neoplasms. In the event of secondary cystic change, the absence of USP6 gene rearrangement and/or the detection of a genetic aberration characteristic of the underlying tumor, such as GNAS SNVs in fibrous dysplasia, ${ }^{11}$ a GRM1 structural alteration in chondromyxoid fibroma, and H3.3 alterations in GCT and chondroblastoma, can help in reaching a diagnosis. 


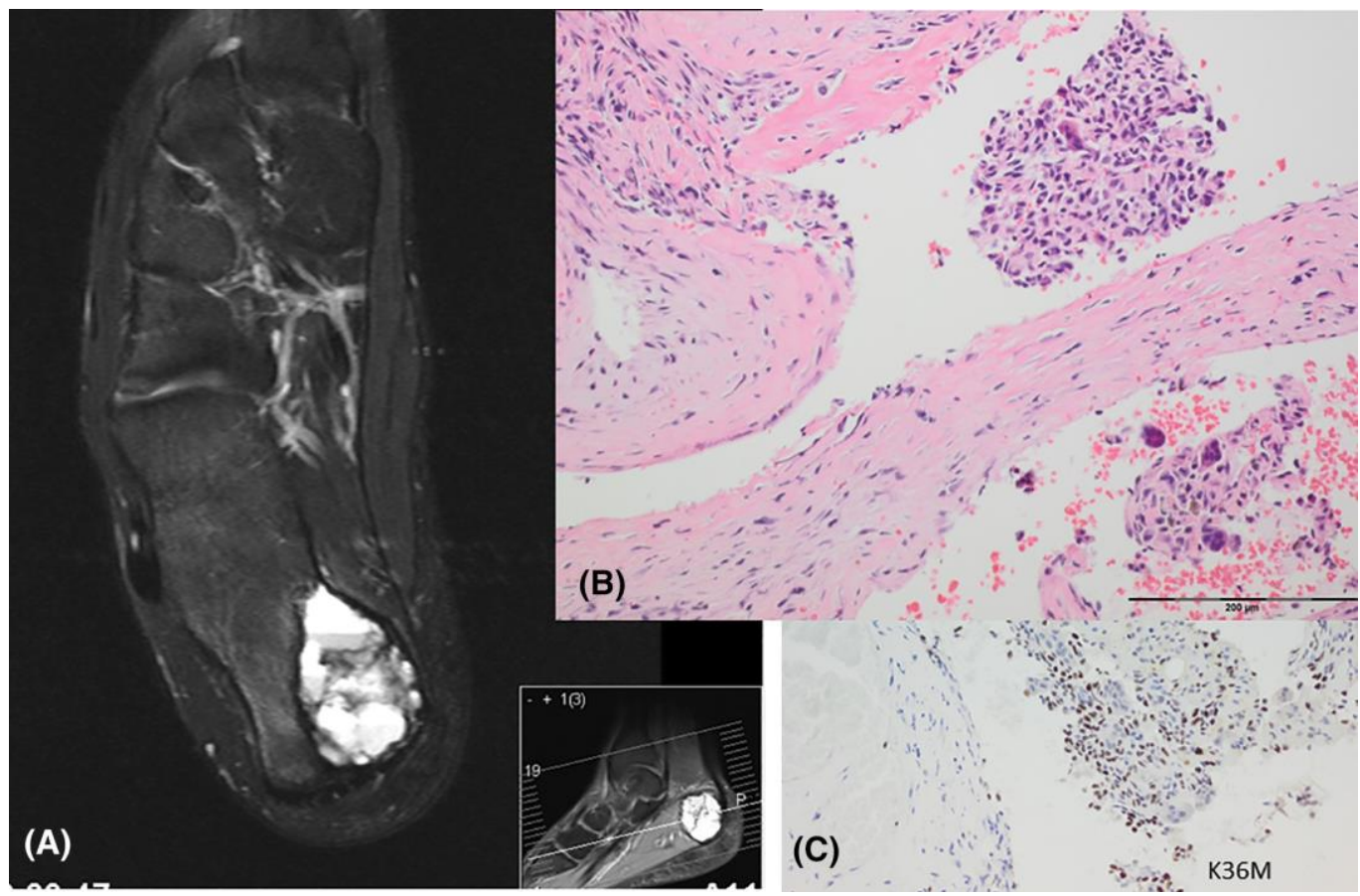

FIGURE 3 A, Axial MRI scan (T2-weighted, TSE, FS) showing a well-defined lobular lytic lesion in the calcaneus with fluid-fluid levels characteristic of aneurysmal bone cyst change. B, Photomicrograph of the curettage specimen showing fibrino-osteoid seams forming the cystic walls along with small fragments of an osteoclast-rich tumor. C, H3.3 K36M immunohistochemistry with nuclear expression highlights the tumor fragments

Detection of a USP6 gene rearrangement is extremely helpful in small samples when the differential diagnosis includes primary $A B C$ and telangiectatic osteosarcoma: two neoplasms with significantly different clinical courses and require distinct treatments.

USP6 rearrangements have also been detected in close to $90 \%$ of nodular fasciitis, a soft tissue tumor, often thought to represent a reaction to trauma that may resolve spontaneously. ${ }^{36,40}$ Although MYH9, on chromosome 22q12.3, is the common fusion partner $(65 \%$ of cases) with USP6 in nodular fasciitis, it has not been reported in $A B C$. The USP6 alteration has also been detected in cases as myositis ossificans, giant cell lesions of small bones, and fibro-osseous pseudotumors of digits suggesting that all such lesions are part of a spectrum of tumors with overlapping histological features. ${ }^{41,42}$

\section{2 | TENOSYNOVIAL GCT}

Tenosynovial GCT, which in its diffuse form is also known as pigmented villonodular synovitis, is a locally aggressive tumor harboring a specific translocation resulting in high levels of colony-stimulating factor 1 expression. ${ }^{43-45}$ Although arising in the synovium of the joint or on the synovial lining of a tendon sheath, it can erode and even destroy the adjacent bone, and in more advanced disease it can be difficult to determine the original site of the tumor. Histologically it can mimic GCT. As the number of the neoplastic cells in the lesion is small, the fusion gene is usually difficult to detect by FISH. However, the test is rarely required as in most cases the histology is diagnostic. Detection of H3.3 p.G34W expression by immunohistochemistry would exclude diagnosis of tenosynovial giant cell tumour as this is restricted to GCT. Tyrosine kinase inhibitors targeting the colonystimulating factor 1 receptor can induce a response in affected patients, therefore making an accurate diagnosis even more important. 46

\section{3 | CHORDOMA}

Chordoma is a primary malignant bone tumor showing notochordal differentiation and is sited along the skeletal axis occurring in bones from the skull base to the coccyx. ${ }^{47}$ Very rarely tumors occur in soft tissues. ${ }^{44,45}$ Chordoma can present at any age from birth to late old age, but most commonly in middle age. It is rarely seen in the black African population. ${ }^{48}$ The median survival is 7 years and it behaves in a locally aggressive manner with metastases occurring typically late in the course of thedisease.

Brachyury (TBXT) expression detected by immunohistochemistry is a highly specific and sensitive biomarker for chordoma: it decorates diffusely and strongly the nuclei of all chordomas other than the rare dedifferentiated variant (Figure 4). Less specific markers include cytokeratins, S100 protein, and aldo-ketoreductase 1B10. ${ }^{47}$ The TBXT protein expression is associated with somatic copy number gains in $27 \%$ of cases which may be seen as just one extra signal on FISH, representing a simple tandem duplication although the genomic events may be more complex. ${ }^{49}$ No pathogenic somatic SNVs in TBXT in chordoma have been reported to date. Nevertheless, evidence for TBXT being implicated in the pathogenesis of the disease is substantial: at a genomic level, germ line tandem duplication of TBXT is a key genetic predisposition event in familial chordoma, ${ }^{50}$ silencing of the 


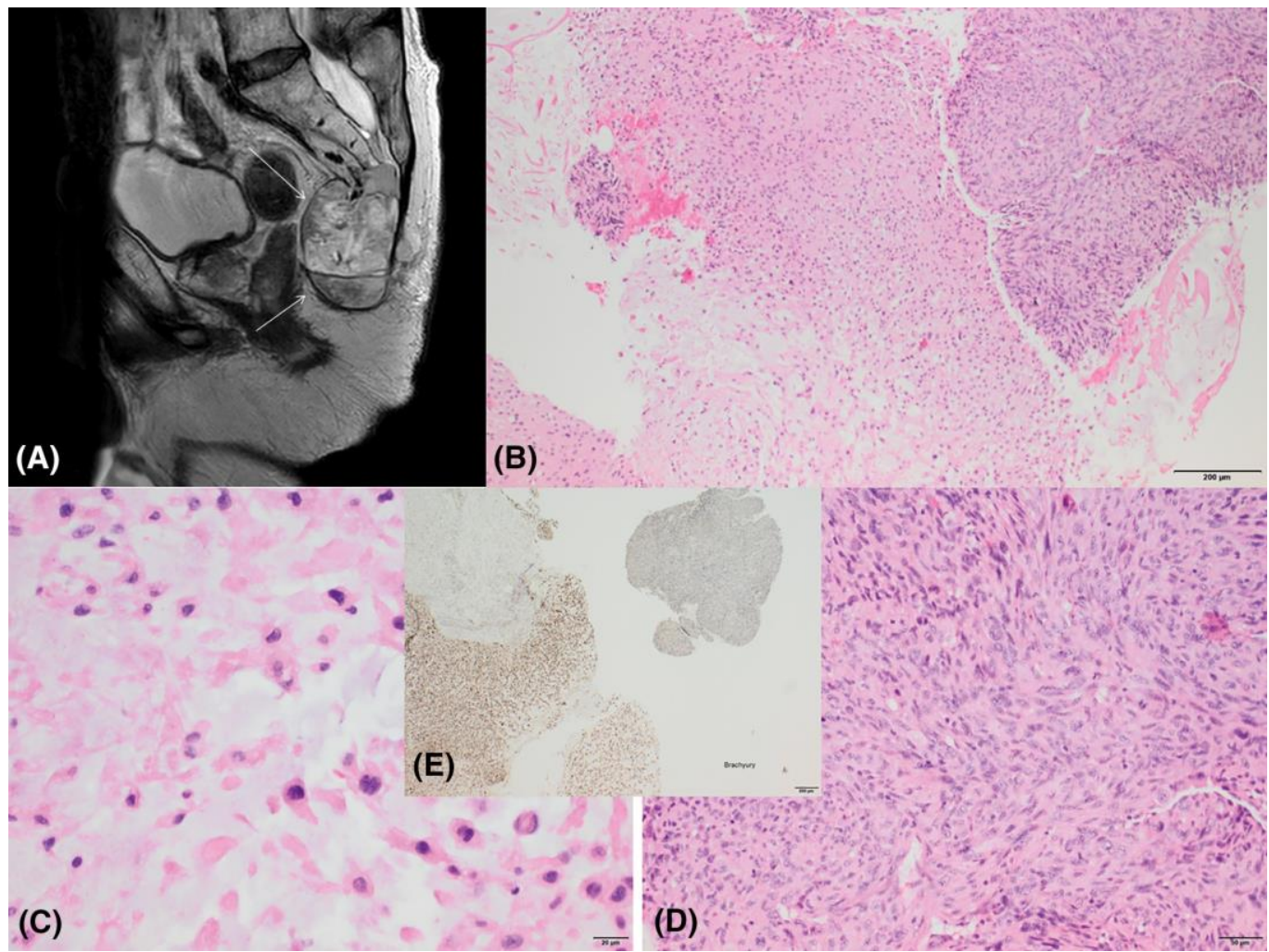

FIGURE 4 A, Sagittal T2W TSE MRI scan showing a large sacral tumor. Photomicrographs showing both conventional (left side) and dedifferentiated chordoma components in one slide (B) and separately (C, conventional; andD, dedifferentiated). Immunohistochemistry for TBXT showing nuclear expression in the conventional areas (left side). E, The top-right dedifferentiated fragment shows no immunoreactivity

gene in chordoma cell lines results in senescence and cell death, ${ }^{51,52}$ and $98 \%$ of patients with sporadic chordoma harbor the rs 2305089 SNP within the DNA binding domain of TBXT. ${ }^{53}$

Whole genome and RNA-sequencing of chordomas identified occasional rearrangements and copy number changes, including chromothripsis, but recurrent gene fusions were not observed. ${ }^{49}$ CDKN2A was confirmed as being a key cancer gene in chordoma supporting the loss of expression in $~-80 \%$ of cases. ${ }^{54}$ As a consequence, CDK4/ CDK6 signaling becomes constitutively activated and might serve as a therapeutic target. ${ }^{55}$ Clinically actionable PI3K signaling mutations including PIK3CA, PIK3R1, and PTEN have been identified in $16 \%$ of cases, although clinical trials are required to determine the clinical value of such targeted therapies. Although EGFR mutations have not been identified inchordomas, the phosphorylated protein is expressed in the majority of cases, and therapeutic compounds have been found to induce chordoma cell death in vitro. ${ }^{56-58}$ This research has resulted in the opening of a recent phase II clinical trial using Afatinib, a third generation EGFR inhibitor. Finally, cancer driver events have also been identified in chromatin remodeling genes including SETD2, $A R I D 1 A$, and $P B R M 1$ raising the possibility that chordoma may be susceptible to epigenetic inhibitors. ${ }^{49,59,60}$

Poorly differentiated chordoma was first described by Mobley et al as tumors with cohesive epithelioid morphology, marked pleomorphism, and mitotic activity. They express TBXT and cytokeratins but unlike conventional chordoma additionally reveal loss of SMARCB1 expression on immunohistochemistry. ${ }^{61}$ The loss of
SMARCB1 expression is due to the frequent homozygous SMARCB1 deletions, which are easily detected by FISH. This subtype of chordoma is most commonly seen in patients under the age of 30 occurring most commonly at the skull base or high cervical vertebra, although more recently a small number have been reported in the sacral region. These tumors typically show an inferior prognosis compared to conventional chordoma. ${ }^{62,63}$

In a recent study, we reviewed 359 chordoma cases, all of which were immunoreactive for TBXT and cytokeratins, for the expression of SMARCB1. Ninety two (25.6\%) of these occurred at the skull base and $57(62.0 \%)$ affected young patients ( $\leq 30$ years old). Four tumors (1.11\%) showed absence of SMARCB1 immunoreactivity. However, the incidence of SMARCB1-negative chordomas reaches 7\% (4 of 57) if only patients younger than 30 years of age presenting with cervical/skull base chordromas are considered "personal unpublished communication."

The identification of the SMARCB1-negtive chordoma subtype is clinically relevant as Enhancer of Zeste homologue 2 (EZH2) inhibitor drugs, such as Tazemetostat, potentially have a therapeutic benefit. Notably five chordomas considered to represent conventional chordomas have been reported to show loss of INI-1 expression. ${ }^{64,65}$ This may reflect the challenge of distinguishing conventional chordoma with atypia and poorly differentiated chordoma on a hematoxylin and eosinstained section, but irrespective of the reason, these cases highlight the importance of assessing the SMARCB1 immunoreactivity status in cases other than those with significant histological pleomorphism as it may 


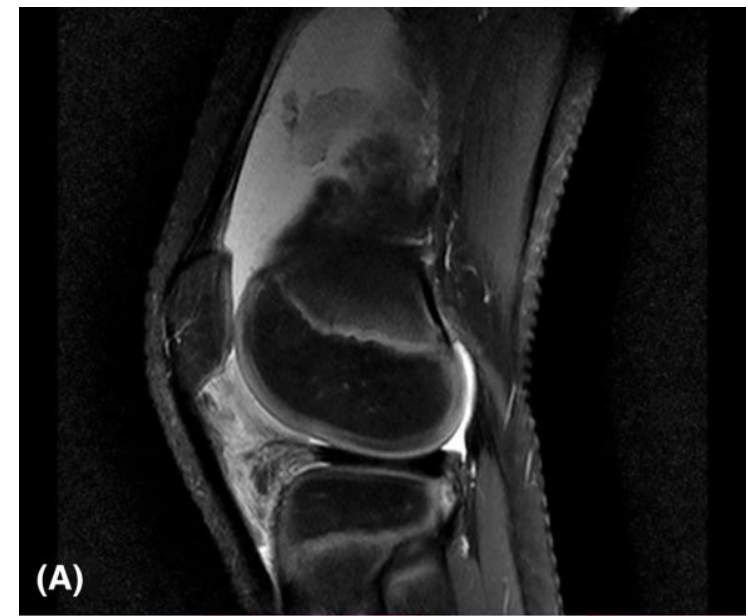

(B)

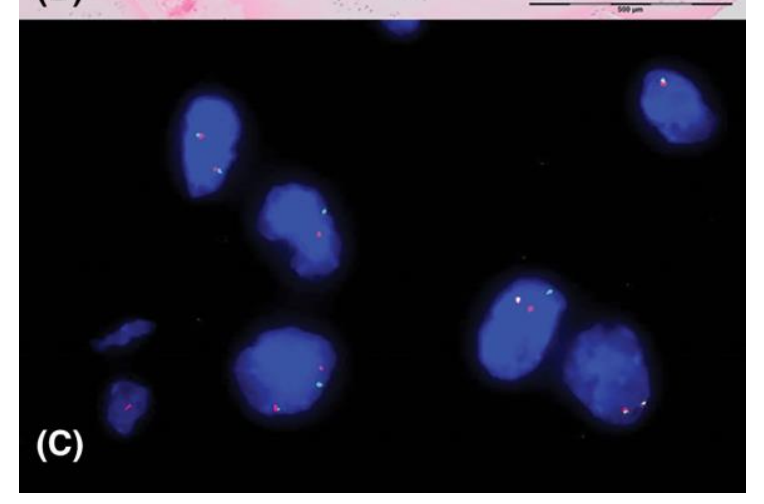

FIGURE 5 A, Sagittal MRI scan of the knee showing nodules within Hoffa's fat pad and lying loose in the superior synovial compartment. B, Photomicrograph of a multinodular bland cartilaginous tumor covered by synovium, consistent with synovial chondromatosis. C, Interphase fluorescence in situ hybridization (FISH) using FN1 break apart probe confirming a FN1 gene rearrangement (split red and green signal in the nuclei)

provide an opportunity for a patient to beentered into a clinical trial for a disease where there are currently only few therapeutic options.

\section{CARTILAGINOUS TUMORS}

Conventional cartilaginous tumors are the most common primary bone tumors and the incidence is likely to be underestimated as many of the benign lesions, enchondromas (central), and osteochondromas (surface) are detected incidentally. Chondrosarcoma is the second most common form of primary malignant bone tumor overall, but is the most common form in adults. The general view is that enchondromas and osteochondromas represent the precursor lesion of central and peripheral chondrosarcoma, respectively, but that transformation occurs in a minority of cases, particularly in the latter. ${ }^{66-68}$

\section{1 | Isocitrate dehydrogenase 1 (IDH1) and isocitrate dehydrogenase 2 mutations}

Isocitrate dehydrogenase 1 (IDH1) and IDH2 somatic, heterozygous, missense, and point mutations were first described in low-grade gliomas, secondary glioblastomas $(80 \%),{ }^{69}$ acute myeloid leukaemia $(16 \%),{ }^{70}$ and less commonly in other neoplasms. The most common mutations include R132 in IDH1 as well as R172 and R140 in IDH2, which all result in changing key arginine residues required for enzyme binding to the substrate isocitrate at the active sites. The mutant enzymes lose the ability to convert isocitrate to $\alpha$-ketoglutarate, and additionally gain a new function that leads to the accumulation of D-2-hydroxyglutarate, ${ }^{71}$ which competitively inhibits a-ketoglutaratedependent enzymes such as histone and DNA demethylases (for review, see Ref. 71).

Sixty percent of central conventional and dedifferentiated as well as periosteal cartilaginous tumors harbor IDH1 R132 or IDH2 R172 (the R140 variant is not reported in cartilage tumors) although the former represents roughly $90 \%$ of all mutations. ${ }^{66,72}$ These IDH1-mutant and IDH2-mutant tumors can be distinguished from their wild-type variants by their methylation profiles. ${ }^{73}$

There are several different $I D H 1$ substitutions at residue p.R132, the most common being R132C occurring in $~ 40 \%$ of IDH-mutant cartilaginous tumors, the other include R132G, R132H, R132L, R132S, R132I, R132Q, and R172S. This is in contrast to IDH-mutant brain tumors of which at least $70 \%$ harbor a $\mathrm{R} 132 \mathrm{H}$ mutation and for which there is an excellent antibody for diagnostic use. IDH1 and IDH2 mutations can occur in tumors of any site but are found more commonly in the tubular bones of the hands and feet with $90 \%$ of these revealing a mutation compared to $53 \%$ of tumors in the long bones of the appendicular skeleton, and $35 \%$ of those in the flat bones. ${ }^{66,74}$ These mutations have never been detected in other types of cartilaginous tumors including osteochondromas, and peripheral (secondary to osteochondroma) chondrosarcomas which harbor a mutation in one of the EXT genes, ${ }^{67}$ clear cell chondrosarcoma, mesenchymal chondrosarcoma, synovial chondromatosis, chondromyxoid fibroma, and chondroblastoma. ${ }^{35,75,76}$ Specifically, these mutations have not been detected in osteosarcoma, making the detection of either an IDH1 or IDH2 mutation a valuable biomarker for distinguishing chondroblastic osteosarcoma from high-grade chondrosarcoma and dedifferentiated chondrosarcoma exhibiting osteosarcomatous differentiation. ${ }^{66}$

IDH1 and IDH2 mutations do not correlate with grade of cartilaginous tumors: the mutations represent early events, and are retained through the life of the tumor, that is, as a tumor progresses from low to high grades and into a dedifferentiated phenotype, in local recurrences and in metastatic lesions. However, neither their presence, nor the different $I D H 1$ mutations at residue p.R132, of which there are several, appear to impact on clinical outcome. ${ }^{66,77}$ This is in contrast 
with IDH1 mutations in glioblastomas which confer a better prognosis compared with $I D H$ wild-type braintumors.

\section{2 | Whole genome and exome sequencing}

Our exome sequencing study of chondrosarcoma showed that the somatic mutation burden in these tumors have a significant association with increasing grade: high-grade chondrosarcomas (grade II, grade III, and dedifferentiated) have on average more than double the somatic mutations per sample as grade I chondrosarcoma. Furthermore, the results confirmed some of what was already known: $33 \%$ of chondrosarcoma harbor alterations in the RB1 pathway, including CDK4, CDK6, and CDKN2A mutations thereby confirming previous reports that loss of $C D K N 2 A$ is a recurrent event in high-grade chondrosarcoma, and is not seen in low-grade disease. ${ }^{78,79}$

Other mutated genes that are commonly found in chondrosarcoma include TP53 (20\%), and genes involved in the hedgehog signaling pathway (18\%) including PTCH1, SUFU, and GLI1 along with RUNX2 and HHIP, some of which represent therapeutic targets. In addition, mutations of other known cancer genes include SETD2, KDM6A, NF2, SF3B1, TET2, DNMT3A, and TSC1.

A novel recent finding in conventional cartilaginous tumor was the presence of $C O L 2 A 1$ in $37 \%$ of cases independent of the presence of $I D H 1 / 2$ mutations; the gene shows hypermutability and the range of mutations consisted of splice site, indels, missense, and large-scale rearrangements. No synonymous mutations were identified. The patterns of mutation were consistent with selection for variants likely to impair normal collagen synthesis. These alterations in COL2A1 appear to be specific to chondrosarcoma. ${ }^{72}$

\section{3 | Synovial chondromatosis}

FN1-ACVR2A and ACVR2A-FN1 in-frame fusions were identified in two cases of chondrosarcoma arising on the background of synovial chondromatosis using whole genome and exome sequencing. Our group reported that 31 of 57 cases (54\%) of synovial chondromatosis and 2 of 3 cases of synovial chondrosarcoma harbor FN1 and/or ACVR2A gene rearrangements as assessed by $\mathrm{FISH}^{80}$ (Figure 4). These alterations define this tumor type but cannot aid in differentiating between benign and malignant forms.

\section{5 | MULTIPLE ENCHONDROMAS (AKA ENCHONDROMATOSIS)}

There are several different forms of enchondromatosis characterized clinically by different phenotypes, some of which are classified genetically. ${ }^{81}$

\section{1 | Ollier disease}

Ollier disease is the most common form of multiple enchondromas and tumors in $80 \%$ of individuals diagnosed clinically with this condition harbor either an IDH1 (98\%) or IDH2 (2\%) heterozygous somatic mutation, and each tumor in an individual carries the same mutation. There is a highly informative conditional knock-in mice demonstrating that failure of cartilage to mature into bone in the growth plate results in persistent small enchondroma-type nodules in the medullary bone. ${ }^{82}$ The mosaic pattern of disease is explained by an early postzygotic (somatic) mutation. The timing of these events is also likely to explain the range of tumors seen in patients with Ollier disease: these include enchondromas and spindle cell hemangiomas in Maffucci syndrome, and the more diverse range of tumors which include gliomas, acute myeloid leukaemia, multiple cartilaginous neoplasms, and hemangiomas, which present in some patients. ${ }^{74,83}$

\section{2 | Less common forms of multiple enchondromas}

Individuals with a rare familial form of an Ollier-type phenotype have been reported as harboring parathyroid hormone receptor 1 mutations. ${ }^{84}$ Metachromatosis is an autosomal dominant disease, characterized by a combination of exostosis and enchondromatosis tumor syndrome, and caused by heterozygous loss-of-function PTPN11 mutations. ${ }^{85}$ Dysspondyloenchondromatosis is inherited as an autosomal dominant trait caused by heterozygous mutations in COL2A1. This syndrome is characterized by short stature with unequal limb length, multiple metaphyseal and diaphyseal enchondromas in the long tubular bones, and osteopenia. ${ }^{86}$ Spondyloenchondrodysplasia is an immuno-osseous dysplasia caused by biallelic mutations in ACP5. ${ }^{87}$

\section{3 | Circulating tumor DNA for IDH1 and IDH2 mutations}

A pilot study has shown that detection of $I D H 1$ or $I D H 2$ mutant molecules is associated with high-grade chondrosarcoma and a worse prognosis. ${ }^{88}$

\section{6 | NONCONVENTIONAL CARTILAGINOUS TUMORS}

\section{1 | Chondromyxoid fibroma}

This benign tumor accounts for $<1 \%$ of bone tumors, generally presents in the metaphysis of long bones, and exhibits a combination of chondroid, myxoid, and fibrous tissue components organized in a pseudolobulated fashion. It may contain atypical cells suggesting malignancy. However, it has never been reported to transform into a high-grade tumor or metastasise. ${ }^{89}$

Whole-genome mate-pair sequencing and RNA sequencing demonstrated that the recombination of the glutamate receptor gene (GRM1) with several $5^{0}$ partner genes, which represent strong promoters, is responsible for the high expression of GRM1 in $90 \%$ of cases. ${ }^{76}$ However, an antibody is not available for diagnostic purposes. This work confirmed the previous report of chromosomal rearrangement of $6 \mathrm{q} 24$, where GRM1 is located. ${ }^{90}$

\section{2 | Clear cell chondrosarcoma}

This nonconventional epiphyseal-based cartilaginous tumor is considered to be a low-grade neoplasm. It has a distinctive morphology with 
chondrocytes resembling hypertrophic cells of the growth plate along with osteoid differentiation leading some to consider that this tumor may be better classified as a variant of osteosarcoma. There are no published next generation sequencing studies on this tumor type. In view of the two other epiphyseal-based tumors, GCT and chondroblastoma, which both harbor histone 3.3 mutations, we looked for these mutations by immunohistochemistry also in clear cell chondrosarcoma and found that 1 of 10 was immunoreactive for p.K36M, a finding confirmed by genotyping. ${ }^{35}$ A consortium from the International Skeletal Society is currently undertaking a review of a large series of cases to address the frequency of histone 3.3 p.K36M mutation in this tumortype.

\section{7 | MESENCHYMAL CHONDROSARCOMA}

This tumor can occur in both bone and soft tissue and exhibits a biphasic appearance including a chondro-osseous, and a small, blue, round cell component. The latter can lead to it being difficult to distinguish from Ewing sarcoma, chondrosarcoma, and osteosarcoma. A HEY1-NCOA2 fusion representing an in-frame fusion of HEY1 exon 4 to NCOA2 exon 13 was identified using exon array profiling. It was found to be highly sensitive being detected in $100 \%$ of cases and also specific for this tumor type, as it has not been detected in other types of chondrosarcoma and Ewing sarcoma. ${ }^{91}$

\section{8 | TUMORS OF UNCERTAIN LINEAGE}

Phosphaturic mesenchymal tumor (PMT) is a neoplasm that arises in bone and soft tissue and frequently gives rise to hypophosphatemic vitamin-D resistant osteomalacia. The most common histological variant is referred to as PMT-mixed connective tissue type. The tumor is composed of spindled to stellate cells in a myxoid/myxo-hyaline matrix. There is usually an associated adipocytic component and grungy-type stromal calcification.

A fibronectin 1 (FN1)-FGFR1 fusion gene and less frequently a FN1-FGF1 fusion are detected in PMT in $42 \%$ and $6 \%$ of cases, respectively demonstrating the central role of the FGF1-FGFR1 signaling in this tumor. The production of fibroblastic growth factor 23 by the tumor cells is the major factor responsible for the oncogenic osteomalacia as it causes hypophosphatemia, hyperphosphaturia, and increased levels of alkaline phosphatase. Neither FGF23 nor FGFR1 immunohistochemistry are helpful for reaching a diagnosis as they are not specific for this disease. Therefore, detection of these fusion gene rearrangements by FISH is to date the most relevant ancillary test. ${ }^{92,93}$

Osteofibrous dysplasia is an unusual primary fibrous osseous bone tumor and is characterized by scattered epithelial cells; it presents in children in most cases, almost exclusively in the tibia and fibula. It can either be inherited as an autosomal trait or occur sporadically. ${ }^{94}$

Massive parallel sequencing of the exomes of a discovery set of four affected members of two families with bilateral disease revealed autosomal dominant germ line mutations in the gene encoding the receptor tyrosine kinase MET that specifically disrupts the differentially spliced exon 14, resulting in functional disruption of the MET receptor. DNA sequencing of lesional tissue from 20 sporadic cases of osteofibrous dysplasia failed to identify similar aberrations of exon 14 splicing. This may reflect the low neoplastic cell population in these tumors which is supported by the work of Gray et al who on studying an additional sample expanded in culture which they then subjected to exome sequencing identified a somatic missense mutation in exon 14, c.3008A>C (p.Tyr1003Ser). This suggests that this MET mutation may represent a common event in the sporadic cases. However, further work is required to establish this. These findings provide opportunities for targeted therapies involving MET inhibitor drugs. ${ }^{95}$

\section{REFERENCES}

1. Baruffi MR, Volpon JB, Neto JB, Casartelli C. Osteoid osteomas with chromosome alterations involving 22q. Cancer Genet Cytogenet. 2001; 124(2):127-131.

2. Nord KH, Nilsson J, Arbajian E, et al. Recurrent chromosome 22 deletions in osteoblastoma affect inhibitors of the Wnt/beta-catenin signaling pathway. PLoS One. 2013;8(11):e80725.

3. Fittall MW, Mifsud W, Pillay N, et al. Recurrent rearrangements of FOS and FOSB define osteoblastoma. Nat Commun. 2018;9(1):2150.

4. Antonescu CR, Chen HW, Zhang L, et al. ZFP36-FOSB fusion defines a subset of epithelioid hemangioma with atypical features. Genes Chromosomes Cancer. 2014;53(11):951-959.

5. Huang SC, Zhang L, Sung YS, et al. Frequent FOS gene rearrangements in epithelioid hemangioma: a molecular study of 58 cases with morphologic reappraisal. Am J Surg Pathol. 2015;39(10):1313-1321.

6. Hung YP, Fletcher CD, Hornick JL. FOSB is a useful diagnostic marker for pseudomyogenic hemangioendothelioma. Am J Surg Pathol. 2016.

7. Behjati S, Tarpey PS, Haase K, et al. Recurrent mutation of IGF signalling genes and distinct patterns of genomic rearrangement in osteosarcoma. Nat Commun. 2017;8:15936.

8. Chen KS, Kwon WS, Kim J, et al. A novel TP53-KPNA3 translocation defines a de novo treatment-resistant clone in osteosarcoma. Cold Spring Harb Mol Case Stud. 2016;2(5):a000992.

9. Collins MT, Singer FR, Eugster E. McCune-Albright syndrome and the extraskeletal manifestations of fibrous dysplasia. Orphanet I Rare Dis. 2012;7(Suppl 1):S4.

10. Delaney D, Diss TC, Presneau N, et al. GNAS1 mutations occur more commonly than previously thought in intramuscular myxoma. Mod Pathol. 2009;22(5):718-724.

11. Idowu BD, Al-Adnani $M$, O'Donnell $P$, et al. A sensitive mutationspecific screening technique for GNAS1 mutations in cases of fibrous dysplasia: the first report of a codon 227 mutation in bone. Histopathology. 2007;50(6):691-704.

12. Lee $\mathrm{SE}$, Lee EH, Park H, et al. The diagnostic utility of the GNAS mutation in patients with fibrous dysplasia: meta-analysis of 168 sporadic cases. Hum Pathol. 2012;43(8):1234-1242.

13. Heim-Hall JM, Williams RP. Liposclerosing myxofibrous tumour: a traumatized variant of fibrous dysplasia? Report of four cases and review of the literature. Histopathology. 2004;45(4):369-376.

14. Carter JM, Inwards CY, Jin L, et al. Activating GNAS mutations in parosteal osteosarcoma. Am J Surg Pathol. 2014;38(3):402-409.

15. Salinas-Souza C, De Andrea C, Bihl M, et al. GNAS mutations are not detected in parosteal and low-grade central osteosarcomas. Mod Pathol. 2015;28(10):1336-1342.

16. Zhang J, Walsh MF, Wu G, et al. Germline mutations in predisposition genes in pediatric cancer. N Eng/ J Med. 2015;373(24):2336-2346. 
17. Daroszewska A, Ralston SH. Genetics of Paget's disease of bone. Clin Sci (Lond). 2005;109(3):257-263.

18. Merchant A, Smielewska $M$, Patel $N$, et al. Somatic mutations in SQSTM1 detected in affected tissues from patients with sporadic Paget's disease of bone. J Bone Miner Res. 2009;24(3):484-494.

19. Camacho-Vanegas O, Camacho SC, Till J, et al. Primate genome gain and loss: a bone dysplasia, muscular dystrophy, and bone cancer syndrome resulting from mutated retroviral-derived MTAP transcripts. Am J Hum Genet. 2012;90(4):614-627.

20. Kovac M, Blattmann C, Ribi S, et al. Exome sequencing of osteosarcoma reveals mutation signatures reminiscent of BRCA deficiency. Nat Commun. 2015;6:8940.

21. Amary F, Ye H, Berisha F, et al. Fibroblastic growth factor receptor 1 amplification in osteosarcoma is associated with poor response to neo-adjuvant chemotherapy. Cancer Med. 2014;3(4):980-987.

22. Behjati S, Tarpey PS, Presneau N, et al. Distinct H3F3A and H3F3B driver mutations define chondroblastoma and giant cell tumor of bone. Nat Genet. 2013;45(12):1479-1482.

23. Amary F, Berisha F, Ye H, et al. H3F3A (histone 3.3) G34W immunohistochemistry: a reliable marker defining benign and malignant giant cell tumor of bone. Am J Surg Pathol. 2017;41(8):1059-1068.

24. Presneau N, Baumhoer D, Behjati S, et al. Diagnostic value of H3F3A mutations in giant cell tumour of bone compared to osteoclast-rich mimics. J Pathol Clin Res. 2015;1(2):113-123.

25. Divisato G, Formicola D, Esposito T, et al. ZNF687 mutations in severe Paget disease of bone associated with giant cell tumor. Am J Hum Genet. 2016;98(2):275-286.

26. Huang L, Teng XY, Cheng YY, Lee KM, Kumta SM. Expression of preosteoblast markers and $\mathrm{Cbfa}-1$ and Osterix gene transcripts in stromal tumour cells of giant cell tumour of bone. Bone. 2004;34(3):393-401.

27. Martin TJ. Historically significant events in the discovery of RANK/RANKL/OPG. World J Orthop. 2013;4(4):186-197.

28. Chawla S, Henshaw R, Seeger L, et al. Safety and efficacy of denosumab for adults and skeletally mature adolescents with giant cell tumour of bone: interim analysis of an open-label, parallel-group, phase 2 study. Lancet Oncol. 2013;14(9):901-908.

29. Sims NA, Martin TJ. Coupling the activities of bone formation and resorption: a multitude of signals within the basic multicellular unit. Bonekey Rep. 2014;3:481.

30. Ikebuchi Y, Aoki S, Honma M, et al. Coupling of bone resorption and formation by RANKL reverse signalling. Nature. 2018;561(7722): 195-200.

31. Frank D, Doenecke D, Albig W. Differential expression of human replacement and cell cycle dependent $\mathrm{H} 3$ histone genes. Gene. 2003; 312:135-143.

32. Lopez-Fernandez LA, Lopez-Alanon DM, Castaneda V, Krimer DB, del Mazo J. Developmental expression of H3.3A variant histone mRNA in mouse. Int J Dev Biol. 1997;41(5):699-703.

33. Schwartzentruber J, Korshunov A, Liu XY, et al. Driver mutations in histone $\mathrm{H} 3.3$ and chromatin remodelling genes in paediatric glioblastoma. Nature. 2012;482(7384):226-231.

34. Wu G, Broniscer A, McEachron TA, et al. Somatic histone H3 alterations in pediatric diffuse intrinsic pontine gliomas and non-brainstem glioblastomas. Nat Genet. 2012;44(3):251-253.

35. Amary MF, Berisha F, Mozela R, et al. The H3F3 K36M mutant antibody is a sensitive and specific marker for the diagnosis of chondroblastoma. Histopathology. 2016;69(1):121-127.

36. Amary MF, Ye H, Berisha F, Tirabosco R, Presneau N, Flanagan AM. Detection of USP6 gene rearrangement in nodular fasciitis: an important diagnostic tool. Virchows Arch. 2013;463(1):97-98.

37. Guseva NV, Jaber O, Tanas MR, et al. Anchored multiplex PCR for targeted next-generation sequencing reveals recurrent and novel USP6 fusions and upregulation of USP6 expression in aneurysmal bone cyst. Genes Chromosomes Cancer. 2017;56(4):266-277.

38. Oliveira AM, Perez-Atayde AR, Dal Cin P, et al. Aneurysmal bone cyst variant translocations upregulate USP6 transcription by promoter swapping with the ZNF9, COL1A1, TRAP150, and OMD genes. Oncogene. 2005;24(21):3419-3426.

39. Oliveira AM, Perez-Atayde AR, Inwards CY, et al. USP6 and CDH11 oncogenes identify the neoplastic cell in primary aneurysmal bone cysts and are absent in so-called secondary aneurysmal bone cysts. Am J Pathol. 2004;165(5):1773-1780.

40. Erickson-Johnson MR, Chou MM, Evers BR, et al. Nodular fasciitis: a novel model of transient neoplasia induced by MYH9-USP6 gene fusion. Lab Invest. 2011;91(10):1427-1433.

41. Bekers EM, Eijkelenboom A, Grunberg K, et al. Myositis ossificans another condition with USP6 rearrangement, providing evidence of a relationship with nodular fasciitis and aneurysmal bone cyst. Ann Diagn Pathol. 2018;34:56-59.

42. Flucke U, Shepard SJ, Bekers EM, et al. Fibro-osseous pseudotumor of digits - expanding the spectrum of clonal transient neoplasms harboring USP6 rearrangement. Ann Diagn Pathol. 2018;35:53-55.

43. de Saint Aubain Somerhausen N, van de Rijn M. Tenosynovial giant cell tumour, diffuse type. In: Fletcher CDM, Bridge JA, Hogendoorn PC, eds. WHO Classification of Tumours of Soft Tissue and Bone. Lyon, France: IARC Press; 2013.

44. Lauer SR, Edgar MA, Gardner JM, Sebastian A, Weiss SW. Soft tissue chordomas: a clinicopathologic analysis of 11 cases. Am J Surg Pathol. 2013;37(5):719-726.

45. Tirabosco R, Mangham DC, Rosenberg AE, et al. Brachyury expression in extra-axial skeletal and soft tissue chordomas: a marker that distinguishes chordoma from mixed tumor/myoepithelioma/parachordoma in soft tissue. Am J Surg Pathol. 2008;32(4):572-580.

46. Gelderblom H, Cropet C, Chevreau C, et al. Nilotinib in locally advanced pigmented villonodular synovitis: a multicentre, open-label, single-arm, phase 2 trial. Lancet Oncol. 2018;19(5):639-648.

47. Flanagan AM, Yamaguchi T. Chordoma. In: Fletcher CDM, Bridge JA, Hogendoorn PC, eds. WHO Classification of Tumours of Soft Tissue and Bone. Lyon, France: IARC Press;2013:328-329.

48. Pan Y, Lu L, Chen J, Zhong Y, Dai Z. Analysis of prognostic factors for survival in patients with primary spinal chordoma using the SEER registry from 1973 to 2014. J Orthop Surg Res. 2018;13(1):76.

49. Tarpey PS, Behjati S, Young MD, et al. The driver landscape of sporadic chordoma. Nat Commun. 2017;8(1):890.

50. Yang XR, Ng D, Alcorta DA, et al. T (brachyury) gene duplication confers major susceptibility to familial chordoma. Nat Genet. 2009;41(11): 1176-1178.

51. Hsu W, Mohyeldin A, Shah SR, et al. Generation of chordoma cell line $\mathrm{JHC7}$ and the identification of Brachyury as a novel molecular target. J Neurosurg. 2011;115(4):760-769.

52. Presneau N, Shalaby A, Ye H, et al. Role of the transcription factor $T$ (brachyury) in the pathogenesis of sporadic chordoma: a genetic and functional-based study. J Pathol. 2011;223(3):327-335.

53. Pillay N, Plagnol V, Tarpey PS, et al. A common single-nucleotide variant in T is strongly associated with chordoma. Nat Genet. 2012;44(11): 1185-1187.

54. Le LP, Nielsen GP, Rosenberg AE, et al. Recurrent chromosomal copy number alterations in sporadic chordomas. PLOS One. 2011;6(5): e18846.

55. von Witzleben A, Goerttler LT, Marienfeld R, et al. Preclinical characterization of novel chordoma cell systems and their targeting by pharmocological inhibitors of the CDK4/6 cell-cycle pathway. Cancer Res. 2015;75(18):3823-3831.

56. Magnaghi P, Salom B, Cozzi L, et al. Afatinib is a new therapeutic approach in chordoma with a unique ability to target EGFR and Brachyury. Mol Cancer Ther. 2018;17(3):603-613.

57. Scheipl S, Barnard M, Cottone L, et al. EGFR inhibitors identified as a potential treatment for chordoma in a focused compound screen. J Pathol. 2016;239(3):320-334.

58. Shalaby A, Presneau N, Ye H, et al. The role of epidermal growth factor receptor in chordoma pathogenesis: a potential therapeutic target. J Pathol. 2011;223(3):336-346.

59. Cottone L, Hookway ES, Cribbs A, et al. Epigenetic inactivation of oncogenic brachyury (TBXT) by H3K27 histone demethylase controls chordoma cell survival. bioRxiv. 2018.

60. Wang L, Zehir A, Nafa K, et al. Genomic aberrations frequently alter chromatin regulatory genes in chordoma. Genes Chromosomes Cancer. 2016;55(7):591-600.

61. Mobley BC, McKenney JK, Bangs CD, et al. Loss of SMARCB1/INI1 expression in poorly differentiated chordomas. Acta Neuropathol. 2010;120(6):745-753. 
62. Cha YJ, Hong CK, Kim DS, Lee SK, Park HJ, Kim SH. Poorly differentiated chordoma with loss of SMARCB1/INI1 expression in pediatric patients: a report of two cases and review of the literature. Neuropathology. 2018;38(1):47-53.

63. Shih AR, Cote GM, Chebib I, et al. Clinicopathologic characteristics of poorly differentiated chordoma. Mod Pathol. 2018;31:12371245.

64. Antonelli M, Raso A, Mascelli S, et al. SMARCB1/INI1 involvement in pediatric chordoma: a mutational and immunohistochemical analysis. Am J Surg Pathol. 2017;41(1):56-61.

65. Yadav R, Sharma MC, Malgulwar PB, et al. Prognostic value of MIB-1, p53, epidermal growth factor receptor, and INI1 in childhood chordomas. Neuro Oncol. 2014;16(3):372-381.

66. Amary MF, Bacsi K, Maggiani F, et al. IDH1 and IDH2 mutations are frequent events in central chondrosarcoma and central and periosteal chondromas but not in other mesenchymal tumours. J Pathol. 2011a; 224(3):334-343.

67. Bovee JV, Cleton-Jansen AM, Wuyts W, et al. EXT-mutation analysis and loss of heterozygosity in sporadic and hereditary osteochondromas and secondary chondrosarcomas. Am J Hum Genet. 1999;65(3): 689-698.

68. Eefting D, Schrage YM, Geirnaerdt MJ, et al. Assessment of interobserver variability and histologic parameters to improve reliability in classification and grading of central cartilaginous tumors. Am J Surg Pathol. 2009;33(1):50-57.

69. Yan H, Parsons DW, Jin G, et al. IDH1 and IDH2 mutations in gliomas. N Engl J Med. 2009;360(8):765-773.

70. Abbas S, Lugthart S, Kavelaars FG, et al. Acquired mutations in the genes encoding IDH1 and IDH2 both are recurrent aberrations in acute myeloid leukemia: prevalence and prognostic value. Blood. 2010;116(12):2122-2126.

71. Dang L, White DW, Gross S, et al. Cancer-associated IDH1 mutations produce 2-hydroxyglutarate. Nature. 2009;462(7274):739-744.

72. Tarpey PS, BehjatiS, Cooke SL, et al. Frequent mutation of the major cartilage collagen gene COL2A1 in chondrosarcoma. Nat Genet. 2013; 45(8):923-926.

73. Guilhamon P, Eskandarpour M, Halai D, et al. Meta-analysis of IDHmutant cancers identifies EBF1 as an interaction partner for TET2. Nat Commun. 2013;4:2166.

74. Amary MF, Damato S, Halai D, et al. Ollier disease and Maffucci syndrome are caused by somatic mosaic mutations of IDH1 and IDH2. Nat Genet. 2011b;43(12):1262-1265.

75. Damato S, Alorjani M, Bonar F, et al. IDH1 mutations are not found in cartilaginous tumours other than central and periosteal chondrosarcomas and enchondromas. Histopathology. 2012;60(2):363-365.

76. Nord $\mathrm{KH}$, Lilljebjorn $\mathrm{H}$, Vezzi F, et al. GRM1 is upregulated through gene fusion and promoter swapping in chondromyxoid fibroma. Nat Genet. 2014;46(5):474-477.

77. Cleven AHG, Suijker J, Agrogiannis G, et al. IDH1 or -2 mutations do not predict outcome and do not cause loss of 5-hydroxymethylcytosine or altered histone modifications in central chondrosarcomas. Clin Sarcoma Res. 2017;7:8.

78. Amary MF, YeH, Forbes $\mathrm{G}$, et al. Isocitrate dehydrogenase 1 mutations (IDH1) and p16/CDKN2A copy number change in conventional chondrosarcomas. Virchows Arch. 2015;466(2):217-222.

79. Schrage YM, Lam S, Jochemsen AG, et al. Central chondrosarcoma progression is associated with pRb pathway alterations: CDK4 downregulation and $\mathrm{p} 16$ overexpression inhibit cell growth in vitro. J Cell Mol Med. 2009;13(9A):2843-2852.

80. Perez-Casanova L, Ye H, Strobl AC, et al. Recurrent FN1 and/or ACVR2A fusion genes are implicated in the pathogenesis of synovial chondromatosis, chondrosarcoma secondary to synovial chondromatosis, and soft tissue chondroma. J Pathol. 2018;246(S1): Abstract 019.

81. Pansuriya TC, Kroon HM, Bovee JV. Enchondromatosis: insights on the different subtypes. Int J Clin Exp Pathol. 2010;3(6):557-569.

82. Hirata M, Sasaki M, Cairns RA, et al. Mutant IDH is sufficient to initiate enchondromatosis in mice. Proc Natl Acad Sci U S A. 2015;112(9): 2829-2834.

83. Pansuriya TC, van Eijk R, d'Adamo $P$, et al. Somatic mosaic IDH1 and IDH2 mutations are associated with enchondroma and spindle cell hemangioma in Ollier disease and Maffucci syndrome. Nat Genet. 2011;43(12):1256-1261.

84. Hopyan S, Gokgoz N, Poon R, et al. A mutant PTH/PTHrP type I receptor in enchondromatosis. Nat Genet. 2002;30(3):306-310.

85. Bowen ME, Boyden ED, Holm IA, et al. Loss-of-function mutations in PTPN11 cause metachondromatosis, but not Ollier disease or Maffucci syndrome. PLoS Genet. 2011;7(4):e1002050.

86. Tran Mau-Them F, Boualam A, Barat-Houari M, et al. Dysspondyloenchondromatosis without COL2A1 mutation: possible genetic heterogeneity. Am J Med Genet A. 2014;164A(3):769-773.

87. Briggs TA, Rice Gl, Adib N, et al. Spondyloenchondrodysplasia due to mutations in ACP5: a comprehensive survey. I Clin Immunol. 2016; 36(3):220-234.

88. Gutteridge A, Rathbone VM, Gibbons R, et al. Digital PCR analysis of circulating tumor DNA: a biomarker for chondrosarcoma diagnosis, prognostication, and residual disease detection. Cancer Med. 2017; 6(10):2194-2202.

89. Romeo S, Aigner T, Bridge JA. Chondromyxoid fibroma. In: Fletcher CDM, Bridge JA, Hogendoorn PC, eds. WHO Classification of Tumours of Soft Tissue and Bone. Lyon, France: IARC Press; 2013: 255256.

90. Romeo S, Duim RA, Bridge JA, et al. Heterogeneous and complex rearrangements of chromosome arm 6q in chondromyxoid fibroma: delineation of breakpoints and analysis of candidate target genes. Am J Pathol. 2010;177(3):1365-1376.

91. Wang L, Motoi T, Khanin R, et al. Identification of a novel, recurrent HEY1-NCOA2 fusion in mesenchymal chondrosarcoma based on a genome-wide screen of exon-level expression data. Genes Chromosomes Cancer. 2012;51(2):127-139.

92. Lee JC, Jeng YM, Su SY, et al. Identification of a novel FN1-FGFR1 genetic fusion as a frequent event in phosphaturic mesenchymal tumour. J Pathol. 2015;235(4):539-545.

93. Lee JC, Su SY, Changou CA, et al. Characterization of FN1-FGFR1 and novel FN1-FGF1 fusion genes in a large series of phosphaturic mesenchymal tumors. Mod Pathol. 2016;29(11):1335-1346.

94. Vigorita VJ, Hogendoorn PC, Sawyer JR. Osteofibrous dysplasia. In: CDM F, Bridge JA, Hogendoorn PC, eds. WHO Classification of Tumours of Soft Tissue and Bone. Lyon, France: IARC Press; 2013:354-355.

95. Gray MJ, Kannu P, Sharma S, et al. Mutations preventing regulated exon skipping in MET cause osteofibrous dysplasia. Am J Hum Genet. 2015;97(6):837-847. 